\title{
On the structure of a reversible entanglement generating set for three-partite states
}

\author{
A. Acín ${ }^{1 *}$, G. Vidal ${ }^{\dagger \dagger}$ and J. I. $\operatorname{Cirac}^{3 \ddagger}$ \\ ${ }^{1}$ GAP-Optique, University of Geneva, 20, Rue de l'École de Médecine, CH-1211 Geneva 4, Switzerland \\ ${ }^{2}$ Institute for Quantum Information, California Institute of Technology, Pasadena, CA 91125, USA \\ ${ }^{3}$ Max-Planck Institut für Quantenoptik, Hans-Kopfermann Str. 1, D-85748 Garching, Germany
}

(July 10, 2006)

\begin{abstract}
We show that Einstein-Podolsky-Rosen-Bohm (EPR) and Greenberger-Horne-Zeilinger-Mermin (GHZ) states can not generate, through local manipulation and in the asymptotic limit, all forms of three-partite pure-state entanglement in a reversible way. The techniques that we use suggest that there may be a connection between this result and the irreversibility that occurs in the asymptotic preparation and distillation of bipartite mixed states.
\end{abstract}

PACS Nos. 03.67.-a, 03.65.Ud

To identify the fundamentally inequivalent ways quantum systems can be entangled is a major goal of quantum information theory. In the case of systems shared by two parties, Alice and Bob, there is only one type of entanglement, namely that contained in the EinsteinPodolsky-Rosen-Bohm (EPR) state

$$
|E P R\rangle=\frac{1}{\sqrt{2}}(|00\rangle+|11\rangle),
$$

in the sense that, in the limit of large $N$, Alice and Bob can reversibly transform $N$ copies of any other state $|\psi\rangle_{A B}$ into EPR states by using only local operations and classical communication (LOCC) [1]. This simple picture becomes much richer in systems shared by more than two parties, since also genuine multipartite entanglement exists [2]. In particular, the Greenberger-Horne-ZeilingerMermin (GHZ) state

$$
|G H Z\rangle=\frac{1}{\sqrt{2}}(|000\rangle+|111\rangle)
$$

can not be reversibly generated from EPR states pairwise distributed among Alice, Bob and a third partie Claire [3]. In the terminology of Ref. [2], this implies that EPR states alone do not form a minimal reversible entanglement generating set (MREGS) for three-partite states.

The results of Ref. [3] left open the question whether, instead, the set

$$
G_{3}=\left\{|G H Z\rangle,|E P R\rangle_{A B},|E P R\rangle_{A C},|E P R\rangle_{B C}\right\}
$$

constitutes a MREGS. Denoting by $\rightleftharpoons$ an asymptotically (i.e. in the large $N$ limit) reversible transformation using LOCC, this question amounts to assessing the feasibility of a transformation of the form

$$
\begin{aligned}
|\psi\rangle_{A B C}^{\otimes N} \rightleftharpoons & |G H Z\rangle^{\otimes g N} \otimes|E P R\rangle_{A B}^{\otimes x N} \\
& \otimes|E P R\rangle_{A C}^{\otimes y N} \otimes|E P R\rangle_{B C}^{\otimes z N}
\end{aligned}
$$

where $g, x, y, z \geq 0$, for any three-partite state $|\psi\rangle_{A B C}$. If this were the case, then entanglement in three-partite systems could be regarded as consisting only of GHZ and EPR correlations.

In the meantime it has been proved that not all fourpartite states can be reversibly generated from a distribution of EPR and three- and four-partite GHZ states 狛. However, no evidence has been found contradicting the following conjecture.

Conjecture: $G_{3}$ is a MREGS for three-partite states.

On the contrary, all reversible transformations of three-partite states so far reported, involving Schmidt decomposable states [2], but also a whole class of more elaborated states [5], seem to support it.

In this Letter we give examples of three-partite states, denoted by $\left|\Psi_{\delta}\right\rangle_{A B C}$, that can not be reversibly generated only with states of the set $G_{3}$, thus disproving the above conjecture. We also show that even a reversible transformation of states of $G_{3}$ into any of these states and states of $G_{3}$ is impossible. That is, we show that there are cases where the transformation of Eq. (州) can not be made reversible even if the coefficients $g, x, y, z$ are eventually allowed to be negative [6. Notice that such a possibility, not previously excluded in four-partite systems, would have allowed for a slightly different description of multipartite entanglement, also based exclusively on EPR and GHZ correlations.

These results, therefore, indicate the need to extend the set $G_{3}$ in order to eventually obtain a MREGS, either in its original formulation or in the extended sense described above. We would like to note, however, that the notion of a non-trivial MREGS implicitly assumes that the manipulation of multipartite pure states can be made reversible. This is, admittedly, an appealing idea, but has not yet been proved. In this sense, our results can be just interpreted as to indicate that a fundamental irreversibility occurs during the process of combining EPR and GHZ entanglements into any of the three-partite pure states $\left|\Psi_{\delta}\right\rangle$.

It is natural to inquire into the origin of such an irreversibility, which is somewhat analogous to the one that 
characterizes the cycle of preparing and distilling bipartite mixed states [7]. Actually, the argument that will lead to disprove the above conjecture would fail if mixedstate entanglement could be reversibly distilled. This fact suggests a connection between the two irreversible processes.

Our strategy consists in showing that a conservation law obeyed in reversible asymptotic entanglement transformations [3] would be violated if EPR and GHZ states could generate $\left|\Psi_{\delta}\right\rangle$ reversibly. Let $|\Psi\rangle_{A B C}$ denote an arbitrary three-partite pure state shared by Alice, Bob and Claire, and let $\rho_{A B}$ be the mixed state resulting from tracing out Claire's subsystem. The relative entropy of entanglement of $\rho_{A B}$ [8],

$$
E_{\Omega}\left(\rho_{A B}\right) \equiv \min _{\sigma_{A B} \in \Omega} S\left(\rho_{A B} \| \sigma_{A B}\right),
$$

where $\Omega$ is some convex set of states (typically, that of separable states) invariant under LOCC and $S(\rho \| \sigma) \equiv$ $\operatorname{tr}\left(\rho \log _{2} \rho-\rho \log _{2} \sigma\right)$ is the quantum relative entropy, was originally introduced to quantify the entanglement of bipartite mixed states. Its regularized version,

$$
E_{\Omega}^{r e g}\left(\rho_{A B}\right) \equiv \lim _{N \rightarrow \infty} \frac{E_{\Omega}\left(\rho_{A B}^{\otimes N}\right)}{N},
$$

is a lower bound for the entanglement cost $E_{c}$ [9, 10] of $\rho_{A B}$, or number of EPR states per copy of $\rho_{A B}$ needed to asymptotically prepare copies of $\rho_{A B}$. It is also an upper bound for its distillable entanglement $E_{d}$ [9, 11], or number of EPR states per copy of $\rho_{A B}$ that can be asymptotically distilled from copies of $\rho_{A B}$. Indeed, $E_{\Omega}^{r e g}$ fulfills the postulates required in 12 for an entanglement measure and therefore 12, 13

$$
E_{c}\left(\rho_{A B}\right) \geq E_{\Omega}^{r e g}\left(\rho_{A B}\right) \geq E_{d}\left(\rho_{A B}\right) .
$$

Particularly relevant in the context of this work will be the fact that, as showed in [3], the relative entropy of entanglement of (say) subsystems $A B, E_{\Omega}(A B)$ must be conserved during any reversible pure-state transformation of the system $A B C$. Applied to transformation (4) this law reads

$$
E_{\Omega}\left(\rho_{A B}^{\otimes N}\right)=E_{\Omega}\left([E P R]_{A B}^{\otimes x N}\right),
$$

$[E P R] \equiv|E P R\rangle\langle E P R|$, where we have used that when tracing out part $C$, only $|E P R\rangle_{A B}$ gives a non-separable contribution [14]. Thus, in the large $N$ limit we are left with the condition

$$
E_{\Omega}^{r e g}\left(\rho_{A B}\right)=x,
$$

where $x$ is the number of EPR states per copy of $\rho_{A B}$ that should be available on the rhs of Eq. (14), and we have used that $E_{\Omega}\left([E P R]_{A B}\right)=1$. Similarly, if instead we allow now for states of $G_{3}$ to appear simultaneously on both sides of transformation (4), we obtain

$$
E_{\Omega}\left(\rho_{A B}^{\otimes N} \otimes[E P R]_{A B}^{\otimes x_{1} N}\right)=E_{\Omega}\left([E P R]_{A B}^{\otimes x_{2} N}\right),
$$

$x_{1}, x_{2} \geq 0$, which implies the condition

$$
\lim _{N \rightarrow \infty} \frac{E_{\Omega}\left(\rho_{A B}^{\otimes N} \otimes[E P R]_{A B}^{\otimes x_{1} N}\right)}{N}=x_{2} .
$$

Now, there are several possible elections of the set $\Omega$. Here we will consider only the set $S e p$ of separable states, and the set $P P T$ of states with positive partial transposition. Each of these choices leads to a different constraint. In particular, Eq. (9) becomes two conditions,

$$
\begin{gathered}
E_{S e p}^{r e g}\left(\rho_{A B}\right)=x, \\
E_{P P T}^{r e g}\left(\rho_{A B}\right)=x .
\end{gathered}
$$

We will next consider pure states $\left|\Psi_{\delta}\right\rangle_{A B C}$ such that its reduced density matrix for systems $A B, \delta$, is a PPT bound entangled state [15], and therefore $E_{P P T}^{r e g}(\delta)=0$. First we will prove that $E_{S e p}^{r e g}(\delta)>0$, which leads to the contradiction $0=x>0$, indicating that $\left|\Psi_{\delta}\right\rangle_{A B C}$ can not be reversibly generated with states of $G_{3}$ [16]. Notice that when applied to the PPT state $\delta$, Eq. (11) for $\Omega=P P T$ implies that $x_{1}=x_{2}$ 17. We will also prove that

$$
\lim _{N \rightarrow \infty} \frac{E_{S e p}\left(\delta^{\otimes N} \otimes[E P R]^{\otimes x_{1} N}\right)}{N}>x_{1},
$$

that by substitution in Eq. (11) for $\Omega=S e p$ implies that $x_{2}>x_{1}$. Therefore, we must have $x_{1}=x_{2}>x_{1}$, which is again a contradiction, this time meaning that the states of $G_{3}$ can not reversibly generate the state $\left|\Psi_{\delta}\right\rangle$ and states of $G_{3}$.

We construct the three-partite states $\left|\Psi_{\delta}\right\rangle_{A B C} \in \mathcal{C}^{d_{A}} \otimes$ $\mathcal{C}^{d_{B}} \otimes \mathcal{C}^{d_{C}}$ as purifications of any PPT bound-entangled state $\delta$ in $\mathcal{C}^{d_{A}} \otimes \mathcal{C}^{d_{B}}$ with no products vectors in its range, the so-called edge bound entangled states [18]. Examples of these states can be found in Refs. [18, 19]. In order to proceed, we need the following result.

Theorem 1 [20]: Consider a projector $P$ onto a subspace $V$ of $\mathcal{C}^{d_{A}} \otimes \mathcal{C}^{d_{B}}$ that does not contain any product vector. A positive constant $\alpha$ exists such that for all $N \geq 1$,

$$
\max _{\left|a_{N} \otimes b_{N}\right\rangle}\left\langle a_{N} \otimes b_{N}\left|P^{\otimes N}\right| a_{N} \otimes b_{N}\right\rangle \leq \alpha^{N},
$$

where $\left|a_{N} \otimes b_{N}\right\rangle \in \mathcal{C}^{d_{A}^{N}} \otimes \mathcal{C}^{d_{B}^{N}}$ denotes a product state.

Proof: $P$ fulfills the following properties: $(i)$ Since there are no product vectors in $V$, a positive number $\alpha_{1}<1$ exists such that $\left\langle a_{1} \otimes b_{1}|P| a_{1} \otimes b_{1}\right\rangle \leq \alpha_{1}$ for all product vectors [21]. (ii) A positive number $c>0$ exists such that $I+c P$ is separable [22]. Then, the proof proceeds as for the projector $P_{b}$ of Ref. [7] with $\alpha \equiv\left(1+\alpha_{1} c\right) /(1+c)$.

The following theorem provides us with a bound for the relative entropy of entanglement with respect to the 
set Sep and together with theorem 1 is the key to the main result.

Theorem 2: Let $P$ be the projector onto the support of a mixed state $\rho_{A B}$ of a bipartite system $\mathcal{C}^{d_{A}} \otimes \mathcal{C}^{d_{B}}$, let $|a \otimes b\rangle \in \mathcal{C}^{d_{A}} \otimes \mathcal{C}^{d_{B}}$ denote a product vector and let $\beta$ be

$$
\beta \equiv \max _{|a \otimes b\rangle}\langle a \otimes b|P| a \otimes b\rangle
$$

The relative entropy of entanglement with respect to separable states is bounded below by

$$
E_{S e p}\left(\rho_{A B}\right) \geq-\log _{2} \beta
$$

Proof: Let $\sigma_{A B} \in S e p$ be the separable state such that $E_{S e p}\left(\rho_{A B}\right)=S\left(\rho_{A B} \| \sigma_{A B}\right)$. The quantum relative entropy can only decrease under a trace-preserving completely positive map $\mathcal{E} 23$. In particular, let us consider

$$
\mathcal{E}(\tau) \equiv P \tau P+(I-P) \tau(I-P)
$$

We find

$$
\begin{aligned}
& S\left(\rho_{A B} \| \sigma_{A B}\right) \geq S\left(\mathcal{E}\left(\rho_{A B}\right) \| \mathcal{E}\left(\sigma_{A B}\right)\right)= \\
& \operatorname{tr}\left(\rho_{A B} \log _{2} \rho_{A B}-\rho_{A B} \log _{2} P \sigma_{A B} P\right),
\end{aligned}
$$

where in the last step we have used that $\rho_{A B}$ is invariant under $\mathcal{E}$ and that we can ignore the contribution $(I-$ $P) \sigma_{A B}(I-P)$ because its support $I-P$ is orthogonal to $P$. Indeed, notice that for positive operators $N, M_{1}$ and $M_{2}, \log \left(M_{1} \oplus M_{2}\right)=\log M_{1} \oplus \log M_{2}$, and therefore $\operatorname{tr}\left[(N \oplus 0) \log \left(M_{1} \oplus M_{2}\right)\right]=\operatorname{tr}\left(N \log M_{1}\right)$. Define

$$
\begin{aligned}
t & \equiv \operatorname{tr}\left(P \sigma_{A B}\right), \\
\sigma_{A B}^{\prime} & \equiv \frac{1}{t} P \sigma_{A B} P .
\end{aligned}
$$

Then, because $\sigma_{A B}=\sum_{i} p_{i}\left|a_{i} \otimes b_{i}\right\rangle\left\langle a_{i} \otimes b_{i}\right|$ is a separable state, we have that $t \leq \beta$. We finally obtain,

$$
\begin{array}{r}
S\left(\rho_{A B} \| \sigma_{A B}\right) \geq \operatorname{tr}\left(\rho_{A B} \log _{2} \frac{\rho_{A B}}{t \sigma_{A B}^{\prime}}\right)= \\
-\log _{2} t+S\left(\rho_{A B} \| \sigma_{A B}^{\prime}\right) \geq-\log _{2} t \geq-\log _{2} \beta,
\end{array}
$$

where we have used that for positive operators $N, M$ and a positive constant $k, \operatorname{tr}(N \log k M)=\operatorname{tr}(N \log M)+$ $(\operatorname{tr} N) \log k$, and the positivity of the quantum relative entropy 23.

We only need to concatenate theorems 1 and 2 to find that for any edge state $\delta$

$$
E_{S e p}\left(\delta^{\otimes N}\right) \geq-\log _{2} \alpha^{N}
$$

and therefore

$$
E_{S e p}^{r e g}(\delta) \geq-\log _{2} \alpha>0,
$$

which disprove the initial conjecture for $G_{3}$.
Notice that we can use this result and the inequalities (7) to extend the irreversibility proved in [7] to all the edge states. Indeed, we have $0=E_{P P T}^{r e g}(\delta)<E_{S e p}^{r e g}(\delta)$, and both quantities are between the entanglement cost $E_{c}$ and the distillable entanglement $E_{d}$.

Let us move now to prove Eq. (14). We need the following two lemmas.

Lemma 1: Let $P$ be a projector onto a subspace $V$ of $\mathcal{C}^{d_{A}} \otimes \mathcal{C}^{d_{B}}$, and let $|a \otimes b\rangle \in \mathcal{C}^{d_{A}} \otimes \mathcal{C}^{d_{B}}$ be a product state. Then

$$
\max _{|a \otimes b\rangle}\langle a \otimes b|P| a \otimes b\rangle=\max _{|\psi\rangle \in V} \lambda_{1}(\psi)
$$

where $\lambda_{1}(\psi)$ denotes the largest coefficient $\lambda_{i}$ in the Schmidt decomposition of $|\psi\rangle,|\psi\rangle=\sum_{i} \sqrt{\lambda_{i}}\left|u_{i} \otimes v_{i}\right\rangle$, $\lambda_{1} \geq \lambda_{i+1}$.

Proof: For any product vector $|a \otimes b\rangle$, let us define the normalized vector $|\gamma\rangle \in V$ as $P|a \otimes b\rangle / \| P|a \otimes b\rangle \|$. Then

$$
\langle a \otimes b|P| a \otimes b\rangle=|\langle a \otimes b \mid \gamma\rangle|^{2} \leq \lambda_{1}(\gamma)
$$

where in the last step we have used lemma 1 of [24]. Let $\left|\psi^{\prime}\right\rangle$ be the vector for which the maximum in the rhs of Eq. (25) is attained, and let $\sum_{i} \sqrt{\lambda_{i}^{\prime}}\left|u_{i}^{\prime} \otimes v_{i}^{\prime}\right\rangle, \lambda_{i}^{\prime} \geq \lambda_{i+1}^{\prime}$, be its Schmidt decomposition. Then

$$
\max _{|\psi\rangle \in V} \lambda_{1}(\psi)=\lambda_{1}^{\prime}=\left\langle u_{1}^{\prime} \otimes v_{1}^{\prime}|P| u_{1}^{\prime} \otimes v_{1}^{\prime}\right\rangle,
$$

which finishes the proof.

Lemma 2: Let $P$ be a projector onto a subspace $V$ of $\mathcal{C}^{d_{A}} \otimes \mathcal{C}^{d_{B}}$ and let $P_{\Phi}$ be a projector onto a bipartite pure state $|\Phi\rangle \in \mathcal{C}^{d^{\prime}} \otimes \mathcal{C}^{d^{\prime}}$ with Schmidt decomposition $\sum_{i=1}^{d^{\prime}} \sqrt{\lambda_{i}}\left|u_{i}\right\rangle \otimes\left|v_{i}\right\rangle, \lambda_{i} \geq \lambda_{i+1}$. Finally, let $\alpha_{p}$ be

$$
\alpha_{p} \equiv \max _{|a \otimes b\rangle}\langle a \otimes b|P| a \otimes b\rangle
$$

where $|a \otimes b\rangle \in \mathcal{C}^{d_{A}} \otimes \mathcal{C}^{d_{B}}$ denotes a product state. Then,

$$
\max _{|c \otimes d\rangle}\left\langle c \otimes d\left|P \otimes P_{\Phi}\right| c \otimes d\right\rangle=\alpha_{p} \lambda_{1}
$$

where the maximization is made over product vectors $|c \otimes d\rangle \in \mathcal{C}^{d_{A}+d^{\prime}} \otimes \mathcal{C}^{d_{B}+d^{\prime}}$.

Proof: Notice that $P \otimes P_{\Phi}$ projects onto a subspace spanned by vectors of the form $|\psi\rangle \otimes|\Phi\rangle,|\psi\rangle \in V$, and that the largest coefficient $\lambda_{1}$ in a Schmidt decomposition fulfills $\lambda_{1}(\psi \otimes \Phi)=\lambda_{1}(\psi) \lambda_{1}(\Phi)$. Then Eq. (29) follows from lemma 1.

We would like to bound below the relative entropy of entanglement $E_{S e p}$ of

$$
\delta^{\otimes N} \otimes[E P R]^{\otimes M} .
$$

The projector onto its support is given by $P_{\delta}^{\otimes N} \otimes$ $[E P R]^{\otimes M}$, where $P_{\delta}$ is the projector onto the support of $\delta$, and we can use lemma 2 and theorem 1 to obtain 


$$
\max _{|a \otimes b\rangle}\left\langle a \otimes b\left|P_{\delta}^{\otimes N} \otimes[E P R]^{\otimes M}\right| a \otimes b\right\rangle \leq \frac{\alpha^{N}}{2^{M}}
$$

where $(1 / 2)^{M}$ corresponds to $\lambda_{1}\left(E P R^{\otimes M}\right)$. Then we can apply theorem 2 to obtain

$$
E_{S e p}\left(\delta^{\otimes N} \otimes[E P R]^{\otimes M}\right) \geq-N \log _{2} \alpha+M,
$$

which implies Eq. (14). This finishes the proof of the fact that it is not possible to reversibly transform states of $G_{3}$ into any purification of a PPT edge state and states of $G_{3}$.

It would be interesting to understand the mechanisms that lead to this irreversibility. Recall that in the asymptotic limit some non-trivial three-partite states can be reversibly generated from EPR and GHZ states [5]. We ignore which conditions determine whether a three-partite pure-state transformation can be performed in a reversible way. The following two facts suggest, however, that there may be a connection between this question and the irreversibility that takes place during the preparation-distillation cycle of bipartite mixed states:

(i) All known three-partite reversible transformations [2.5] involve pure states whose bipartite reduced mixed states can be distilled and prepared in a reversible way 25.

(ii) The proof that $G_{3}$ is not a MREGS relies on the irreversibility that occurs in bipartite mixed-state manipulation. Indeed, suppose that $E_{c}$ and $E_{d}$ would not disagree for edge states. Then, because of Eq. (7), $E_{P P T}^{r e g}$ and $E_{\text {Sep }}^{r e g}$ would also have been equal, and this would jeopardize our argument.

Finally, a major open question is whether a finite MREGS exists for three-partite states and, if so, which kind of states must include. These are difficult issues that certainly deserve further investigation. We cautiously conclude the present work by noting that the states of an eventual MREGS must have bipartite reduced density matrices able to reproduce the discrepancies between relative entropies displayed by the states $\delta$, and must therefore carry themselves the signature of bipartite mixedstate irreversibility.

A. A. thanks J. Preskill and the IQI, Caltech, for hospitality. We thank D. P. DiVincenzo, W. Dür, E. Jané, N. Linden, Ll. Masanes and S. Popescu for discussion. This work was supported by the European project EQUIP (IST-1999-11053), by the ESF, by the Swiss FNRS and OFES, and by the NSF (of USA), Grant. No. EIA0086038 .

Note added: after completion of this work, Y. Shi pointed out the relation between the results proved here and his recent work [26. We have not been able to follow the line of argumentation in such a work.

\footnotetext{
*Antonio.Acin@physics.unige.ch

†vidal@cs.caltech.edu

${ }^{\ddagger}$ Ignacio.Cirac@mpq.mpg.de
}

[1] C. H. Bennett, H. J. Bernstein, S. Popescu and B. Schumacher, Phys. Rev. A 53 (1996), 2046.

[2] C. H. Bennett, S. Popescu, D. Rohrlich, J. A. Smolin and A. V. Thapliyal, Phys. Rev. A 63 (2001), 012307.

[3] N. Linden, S. Popescu, B. Schumacher and M. Westmoreland, quant-ph/9912039.

[4] S. Wu and Y. Zhang, quant-ph/0004020.

[5] G. Vidal, W. Dür and J. I. Cirac, Phys. Rev. Lett. 85 (2000), 658.

[6] A negative value for, say, the coefficient $x$ of Eq. (4) can be used to symbolize that the states $|E P R\rangle_{A B}$ must appear on the lhs of that transformation. In the present work this possibility is considered as a special case of allowing any state of $G_{3}$ to appear simultaneously and in arbitrary proportions on both sides of Eq. (何).

[7] G. Vidal and J. I. Cirac, Phys. Rev. Lett. 86 (2001), 5803.

[8] V. Vedral, M. B. Plenio, M. A. Rippin and P. L. Knight, Phys. Rev. Lett. 78 (1997), 2275; V. Vedral and M. B. Plenio, Phys. Rev. A 57 (1998), 1619.

[9] C. H. Bennett, D. P. DiVincenzo, J. A. Smolin and W. K. Wootters, Phys. Rev. A 54 (1996), 3824.

[10] P. M. Hayden, M. Horodecki and B. M. Terhal, J. Phys. A 34 (2001), 6891.

[11] E. M. Rains, Phys. Rev. A 60, 173 (1999).

[12] M. Horodecki, P. Horodecki and R. Horodecki, Phys. Rev. Lett. 84 (2000), 2014.

[13] An heuristic justification for the second of these inequalities can be found in M. Plenio and V. Vedral, Contemp. Phys. 39, 431 (1998).

[14] The relative entropy of entanglement fulfills $E_{\Omega}\left(\rho_{A B} \otimes\right.$ $\left.\rho_{s}\right)=E_{\Omega}\left(\rho_{A B}\right)$ for any separable state $\rho_{s}$ as a result of its monotonicity under LOCC [8].

[15] M. Horodecki, P. Horodecki and R. Horodecki, Phys. Rev. Lett. 80 (1998), 5239.

[16] The relation between $(i)$ the equivalence of PPT and separable relative entropies of entanglement and $(i i)$ the question whether $G_{3}$ is a MREGS for three-partite states, has been previously considered by E. F. Galvao, M. B. Plenio and S. Virmani, J. Phys. A 33 (2000), 8809.

[17] For $\rho_{p p t}$ a PPT state we have $E_{P P T}\left(\rho_{A B} \otimes \rho_{p p t}\right)=$ $E_{P P T}\left(\rho_{A B}\right)$, since $(i)$ by means of LOCC we can get rid of $\rho_{p p t}$ and LOCC can only decrease $E_{P P T}$, so that $E_{P P T}\left(\rho_{A B} \otimes \rho_{p p t}\right) \geq E_{P P T}\left(\rho_{A B}\right)$, and (ii) for any PPT state $\pi_{p p t}$ we have $S\left(\rho_{A B} \otimes \rho_{p p t} \| \pi_{p p t} \otimes \rho_{p p t}\right)=$ $S\left(\rho_{A B} \| \pi_{p p t}\right)$, which guarantees that $E_{P P T}\left(\rho_{A B} \otimes \rho_{p p t}\right)$ is not going to be larger than $E_{P P T}\left(\rho_{A B}\right)$.

[18] M. Lewenstein, B. Kraus, J. I. Cirac and P. Horodecki, Phys. Rev. A 62, 52310 (2000).

[19] P. Horodecki, Phys. Lett. A 232 (1997), 333; C. H. Bennett, D. P. DiVincenzo, T. Mor, P. W. Shor, J. A. Smolin and B. M. Terhal, Phys. Rev. Lett. 82 (1999), 5385; D. Bruß and A. Peres, Phys. Rev. A 30 (2000), R30301.

[20] D. P. DiVincenzo, private communication, has pointed out to us this powerful generalization of a result of 7 .

[21] B. M. Terhal, Lin. Alg. Appl. 323 (2000), 61. 
[22] K. Zyczkowski, P. Horodecki, A. Sanpera and M. Lewenstein, Phys.Rev. A 58 (1998) 883.

[23] See for instance M. A. Nielsen and I. L. Chuang, Quantum Computation and Quantum Information, Cambridge University Press (2000).

[24] G. Vidal, D. Jonathan and M. A. Nielsen, Phys. Rev. A 62, 012304 (2000).

[25] This can be checked by noticing that the bipartite reduced density matrices of the states discussed in 5 (which contain the Schmidt-decomposable states of as a particular case) consist of a mixture of locally orthogonal pure states [5] (either product or entangled). Thus, the entanglement of the mixed state can be distilled without losses by means of a projective local measurement that probabilistically picks up one of the pure states of the mixture.

[26] Yu Shi, quant-ph/0201079. 